\title{
An adaptive group decision pattern and its use for industrial security management
}

\author{
Heiko Thimm*, Robert Katura \\ Pforzheim University, School of Engineering, Pforzheim, Germany \\ Email address: \\ Heiko.thimm@hs-pforzheim.de (H. Thimm), Robert.katura@hs-pforzheim.de (R. Katura)
}

\section{To cite this article:}

Heiko Thimm, Robert Katura. An Adaptive Group Decision Pattern and its Use for Industrial Security Management, American Journal of Environmental Protection. Vol. 1, No. 1, 2012, pp. 1-8. doi: 10.11648/j.ajep.20120101.11

\begin{abstract}
In response to critical events Security Management Organizations (SMO) need to follow plans. Among others response plans can require decisions to be made by several SMO people. For security situations with a not too high time pressure it is possible to repeatedly perform a decision making process that includes decision makers that are separated by time and space. The better understanding and new information obtained in a decision process cycle by corresponding adaptations of the decision process and the underlying decision model can be exploited in the next following process cycle. This adaptive group decision pattern can lead to better decision results. In order to not over-challenge a SMO by the extra group coordination and moderation efforts of this pattern one can make use of a Group Decision Support System (GDSS) with special enhancements for this pattern. In this article a respective new group decision pattern is introduced and demonstrated in combination with an enhanced GDSS through a fictive industrial security case example. A process model for security incident management and a process model for adaptive complete asynchronous group decision making are described using the BPMN2.0 graphical process modeling standard. A research prototype of the assumed GDSS that is enhanced to support the new group decision making pattern is currently implemented.
\end{abstract}

Keywords: Hazardous Material Management, Security Incident Management, Group Decision Making, Analytical Hie-rarchy Process (AHP), Process Modeling

\section{Introduction}

It is a common security measure to monitor crucial infrastructures such as power plants, industrial parks, and airports through special equipment such as sensor devices, video cameras, and unmanned flying objects. The monitoring is oriented at "security events" (also referred to as "security incidents") which are defined as observable occurrence(s) in a system or network that violate the security policy of an organization [14]. One major task of Security Management Organizations (SMO) is to check security events and to identify and classify the events in terms of their priority. The priority of events determines the response that is required from the SMO. In many cases the SMO's response handling is based on guidelines and response plans [12][18][5]. These plans are carefully prepared and frequently checked in order to assure their effectiveness and to assure that legal regulations are fulfilled.

The guidelines often require from the SMO personal to collaboratively make, document, and communicate deci-sions [2][7]. In security management situations typical decisions include prioritization decisions such as assessment decisions, diagnosis decisions, selection decisions, and allocation decisions. In general in prioritization decisions one has to determine a specific alternative (i.e. a specific course of action; usually the alternative from which it is expected that it will result into the best or most fitting result) among a given set of alternatives. For example, assume that a too high concentration of hazardous particles in a production hall of a chemical plant has caused a security event. As part of the preplanned response handling it can be requested from the SMO to choose proper response actions. A cor-responding selection decision will present to the decision maker(s) (e.g. production safety engineers, maintenance engineers) a set of response action alternatives. Through the assignment of priorities a ranked list of alternatives can be obtained. The top ranked alternative corresponds to the most appropriate action alternative with respect to the judgements of the decision makers and the available information base.

The performance of single person decisions (individual decisions) in comparison to multi person decisions (group decisions) has been studied by several research groups with different results [1][6][16]. Several studies suggest that 
group decisions can in most cases lead to better decisions. Other investigations obtained the opposite result. There exists however evidence that group decisions are especially useful for solving complex decision problems while individual decisions are more suitable for simple decision problems.

Given a complex security event we assume that the gen-eral benefits of group decisions such as the consideration of more comprehensive background information, expert knowledge and experience, and the power of group judge-ments can be useful for many cases of industrial security management. That group decisions are addressed in today's practical security management guidelines and recommen-dations supports our assumption [7][15].

Today's available Group Decision Support Systems (GDSS) such as Decision Lens, Expert Choice, and nemo2 offer functionalities for effective information sharing, efficient group interaction, standard group decisions methods, and remote decision makers. Forthcoming more enhanced GDSS will offer new support functions for group decision making such as support of mobile decision makers [9] and functions for automated moderation of group decision making processes [17]. Not only will SMO through the use of enhanced GDSS be able to improve effectiveness of their group decision processes. New group decision making patterns will be enabled by properly enhanced GDSS. Especially new patterns are promoted that so far are considered to be too complicated or to require too much attention from a human group moderator.

One particular novel group decision making pattern is the adaptive complete asynchronous group decision pattern proposed in this article where participants are separated by location and time. In this pattern group decision making is defined as an iterative adaptive process that is executed based on a subsequently revised decision model. The adap-tation actions concerning the process and possibly the decision model too are driven by the feedback obtained from the participants. The feedback is used by a human group moderator in order to determine if it is useful to complete the process another time. It is expected that this pattern of group decision making can due to learning and training effects lead to better informed decision making processes and ultimately to better decision results.

A conceptual framework for how the general adaptive group decision pattern can be exploited especially for the purpose of security event management is proposed. The framework is based on the modeling of security event management processes using the standardized BPMN modeling notation [10]. A generic process model for security incident management is described. The incident manage-ment process allows an on duty incident manager to enact and perform an individual group decision making process that is to a large degree managed and controlled by a GDSS.

In the next section different general patterns of group decision making and especially the adaptive group decision pattern are analyzed. The above described case of a chemical company that needs to deal with air pollution in a production plant is subsequently refined as sample case. Section 3 details the proposed adaptive group decision pattern and describes the use of the pattern for industrial security event management. This includes sample process models and a description of how corresponding process instances can be executed based on an enhanced GDSS. The current status of the still ongoing research and concluding remarks are given in the concluding section.

\section{Group Decision Patterns in an In-dustrial Security Case Example}

A common pattern of security management plans is a pattern where first several different courses of actions to handle a given security event and a set of criteria to evaluate these alternatives are specified. Following that a decision model is created that is intended to help the SMO members to identify the best alternative. In order to exploit the ex-pertise and experience of several people a multi-person (or group) decision process can be used. Several researcher groups investigated this approach in the security manage-ment domain including [8][7][3].

In a group decision making process it is expected from the participants to judge the available alternatives in terms of the given criteria. Following that the individual judgments are aggregated to the group decision result. A popular method to obtain the aggregated group result is the Analytical Hierarchy Process (AHP) of Thomas Saaty[13]. With the AHP both quantitative and qualitative evaluation criteria can be combined in a single decision model.

Reconsider the above described sample case of a security incident in a production hall of a chemical plant where a too high concentration of hazardous particles is detected in the air. Let us assume that an appropriate action response is to be determined by a group decision including four participants: 1. the plant safety engineer, 2 . the respective product manager, 3. the team head of the plant maintenance team, 4. a respective person from the cost accounting department. As a possible set of decision alternatives let us consider:

Alternative 1:Continue the production process without changes and check equipment to detect air contamination by hazardous material.

Alternative 2:Continue the production in a reduced and tightly monitored mode, instruct personal to wear extra safety clothing.

Alternative 3:Bring the production subsequently in a predefined pause mode and instruct maintenance engineers to check the facility.

Alternative 4:Shut down production (abruptly) and in-struct maintenance engineers and external specialist to check the facility.

One can consider the following criteria as evaluation criteria to judge these alternatives: possible damage to people, possible damage to equipment, possible damage to quality of production output, cost incurred by a reduced 
production output, disturbance for other areas due to inter-ventions.

Apart from the advantages of a broader expertise and broader information base that is obviously beneficial for decision making in general group decision making can lead to further advantage over single person decisions. With group decisions it is possible to reduce the risk of a bad decision due to the human factors (e.g. the responsible decider can be over-challenged by the decision problem). Also group decisions can lead to better acceptance, loyalty, and motivation by the people involved. Of course, there exist also drawbacks of group decisions such as the needed extra time, a relatively large coordination effort, and the demand for a trained moderator. Furthermore, group decisions can be biased by so-called Group Think Effects [4]. For example, the illusion of invulnerability can occur within a group and create excessive optimism and encourage risk taking. Group decisions in security management scenarios can be obtained in a synchronous mode that is by conducting face-to-face meetings. However, group members can be indispensable somewhere else due to other security management duties or other obligations. Also time or budget restrictions may not allow a personal meeting. For these and other reasons in some security situations the group decision is to be performed in an asynchronous mode. According to the Groupware literature the term "asynchronous" means that some participants are separated at least with respect to one of the two dimensions time and location.

For security management situations dislocated group de-cision participants are not necessarily an unfavorable situation. Often, it can be helpful to consider SMO members that are situated close to the scene and that are equipped with mobile communication devices [9] as remote participants. They can contribute first-hand information and impressions of the incident status to the group decisionprocess.

If we extend the industrial security sample case by the assumption that the incident occurred at a large global chemical corporation then the group decision will possibly include asynchronous participants. For example, assume that the incident occurred at one of the corporation's several production sites in India and that the safety operation is managed centrally by a team of plant safety specialists at the Indian headquarter. Furthermore, presume that the product management team and also the responsible cost accounting team are located at the corporation's global headquarter in Germany. Given this situation the participants of the group decision - plant maintenance team head at the site where the incident happened and three other remote experts in different time zones - are required to perform an asynchronous group decision making process.

Group decisions to select the best course of security management action(s) among a set of alternatives are an interesting subject of research. In our research framework we especially investigate novel Information and Commu-nication Technology solutions for security situations where SMO members are separated by both location and time. We refer to these cases by the notion of complete asynchronous group decisions. The word "complete" is used to signify that the focus is on a dual separation and not on a partial separation.

The pattern of complete asynchronous group decisions is certainly not applicable to all security situations. If a deci-sion is needed and to be carried out urgently in order to prevent severe damages to the environment, humans, and company assets then an asynchronous group decision is not useful due to the extra time needed. For example, if in the sample case the level of air contamination with hazardous particles is a severe thread to the human health then certainly immediate counter actions are required. However, if the concentration level is only slightly increased and is far away from being a thread to the people working in the production hall then it can be appropriate to perform an asynchronous group decision. That is due to a relatively low level of time pressure for a response the most appropriate counter action can be determined by an asynchronous group decision process.

That the final decision is not yet expected to be available and carried out can mean for some security situations that the decision is simply postponed (i.e. it is just waited) and/or other tasks are performed instead of the decision task. An alternative to that is to obtain a decision result in the first place and to consider the option of another repetition of the group decision process instead of carrying out the decision immediately. The option of a process repetition can be useful especially when the security situation has changed in the meantime. Also a repetition can be useful when feedback given by participants of the previous decision cycle can be used for improvements of the information base, the decision model and/or the group decision process. For the sample case let us assume that the alternative 1 - Continue the production process without changes and check equipment to detect air contamination by hazardous material - was determined by an initial group decision process. If the air contamination level is still just slightly more than normal when the decision is made then instead of carrying out the action (i.e. check equipment) it can be useful to once more complete the group decision process within a given time frame.

We call this refined pattern an adaptive complete asyn-chronous group decision process. In the next section the conceptual approach of this pattern and also the potential advantages are described.

\section{Adaptive Group Decision Pattern - Modeling and GDSS-based Execution}

A conceptual overview of the pattern of adaptive com-plete asynchronous group decisions is given in the Figure 1. The difference between this proposed pattern and other group decision patterns is that a repetitive completion of the process is considered. Modifications of the process itself and also the underlying decision model can occur each time before the process is restarted again. However, only 
when there is no need to immediately carry out the decision result a further process repetition is considered to be an

option.

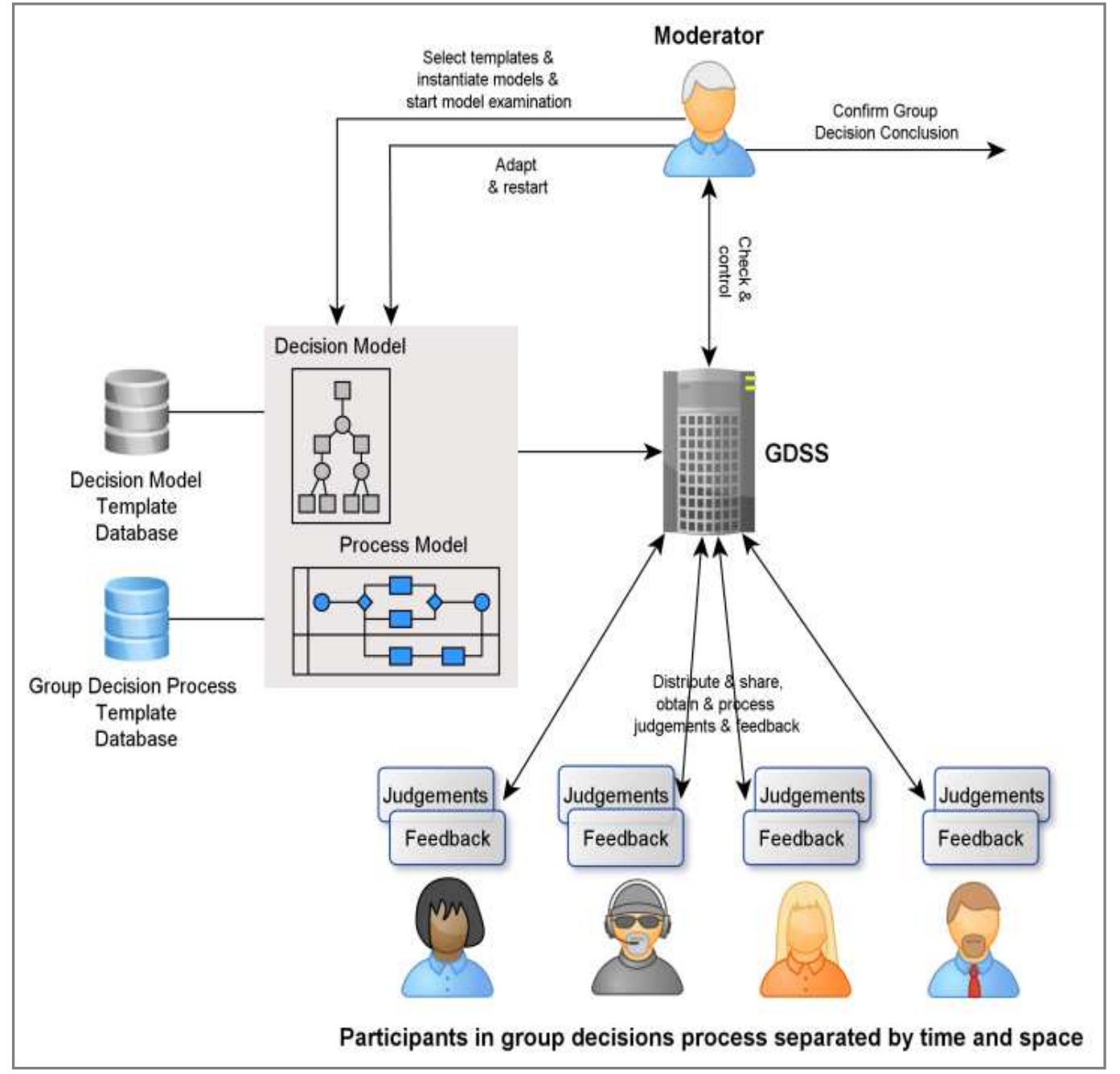

Figure 1.Conceptual model of adaptive complete asynchronous group decision making.

It is the underlying idea that the participants' under-standing of the problem situation can improve with each completion of the decision process and to promote this optimization potential by a corresponding process structure.

This additional understanding gained by the participants can be used as feedback for a further completion of the decision process. Among others the feedback from the participants can include important background information, background knowledge, opinions, judgements, additional decision alternatives, new evaluation criteria, or a proposal for additional group members. The feedback from the participants can be the result of a learning progress, new insights, new ideas inspired by shared information, and other intellectual effects that can emerge by the re-consideration of a decision problem. The use of the feedback can lead to modifications of the information base shared among the participants, modifications of the group decision process, and modifications of the decision model. It is expected that the modifications in general lead to a more complete and more accurate information base, a more accurate and with respect to the current security situation a better fitting decision model and group decision process. Through these improvements it is possible to obtain a better decision result.

For example, reconsider the above described case of an air pollution problem in a chemical plant. Assume that a first group decision result was obtained by an adaptive complete asynchronous group decision process with the alternative 1 as the top ranked alternative. Recall from earlier that alter-native 1 means "Continue the production process without changes and check equipment to detect air contamination by hazardous material". Let us assume that the completion of a first group decision cycle lead to the following two feedback items:

Feedback item 1: The plant safety engineer at the Indian headquarter advises to additionally involve also a specific chief maintenance engineer of another plant into the group decision.

Feedback item 2: The responsible product manager pro-poses to add to the decision alternative 1 the extra measure to check product quality with a double than normal 
checking frequency.

Given this situation and under the assumption that the degree of air pollution has not increased in the meantime it can be useful to accordingly perform changes and to repeat the group decision process again.

Using an analogy from programming languages one can say that adaptive complete asynchronous group decisions are intended to be performed in a loop. In more details the decision process is embraced in a foot-controlled loop with a termination condition at the end (for example: repeat $<$ in-structions $>$ until <termination condition $>$ ). There exist different approaches for the termination condition. For example it is possible to let the group participants decide if the group decision is to be repeated. In the research presented in this article we focus on another alternative where a human group decision moderator makes this decision. Assuming that a group decision moderator has got most complete information about the security situation and the group decision process she/he can decide if it is useful to repeat the group decision process again. Not only is it required to consider for this (termination) decision the level of time pressure of the security situation. It is also required to judge if the expected improvements of the decision are worth the efforts to complete the decision process again. In this context one needs to consider that the repetition of a group decision process can benefit from training effects and thus being completed in less time as compared to former process completions. Furthermore, one also needs to consider that through the use of properly enhanced Group Decision Support Systems (GDSS) the coordination and moderation tasks of group decisions can be effectively supported [17].

Concerning the sample security case the safety engineer at the remote Indian headquarter can act as the group decision moderator in addition to her/his role as usual group decision participant.

It is expected from the SMO and especially the decision moderator and the group participants to fulfillspecial re-quirements in order to obtain the full benefits of the pattern of adaptive complete asynchronous group decisions. These requirements include an effective communication and information sharing among the separated people and an efficient organization, coordination, monitoring, and control of group decision processes. The capability to efficiently exchange, collect, and analyze feedback from participants, and the capability to flexibly adapt group processes and decision models are other key requirements. By the use of a GDSS with corresponding enhancements that address these requirements it is possible for an SMO to effectively handle security events. With respect to the sample case, through a corresponding GDSS such as the GRUPO-MOD System [17] the safety engineer who acts as the group decision moderator can benefit from partially or completely automatically performed activities such as information management activities, moderation activities, and coordination activities.

In the remainder of this section a refined version of the case study is described in which the pattern of adaptive complete asynchronous group decisions is used by the SMO of a chemical plant. In comparison to the so far above described case study the refinement concerns a combined use of i) process modeling techniques and ii) an enhanced GDSS. The development of security management solutions based on techniques from these two areas is the general goal of the research presented in this article.

It is our assumption that one can effectively specify se-curity management processes in general and especially group decision making as part of such processes by the use of the BPMN modeling standard [10]. For the case example of the security incident at the chemical plant two sample process models are described below based on the standard BPMN2.0. The first model shows the general security inci-dent management process of the chemical company. The second model is a generic model for adaptive asynchronous complete group decision making to handle specific types of security incidents. Note that the purpose of especially this second model is to serve as a template (i.e. repeatable pattern) from which one can create individual process instances.

Apart from its wide spread use and large tool support BPMN offers the capability that BPMN models can be mapped into other formats that can be executed in corres-ponding business process execution environments. In our research we obtained first positive indications that this capability of BPMN can be used to enable partially auto-mated group decision making processes in security man-agement scenarios. It is the objective to achieve the partial automation by a GDSS system that is enhanced with special functionalities to create individual process instances (i.e. proxies for real world group decision processes) from process templates and to execute them. When an individual process instance is executed the GDSS performs actions such as the notification of participants, the collection of the participants' decisions, the aggregation of the individual decisions to a corresponding group decision, and also central data management and group moderation tasks (e.g. sending reminders). An example of such an enhanced GDSS is the GRUPO-MOD system presented in [17].

In the following sample process models the general role of enhanced GDSS in security management scenarios is described. It is also shown how enhanced GDSS can effec-tively support adaptive complete group decisions in security management situations.

The model shown in Figure 2 has the following meaning. The big rectangle (called pool in BPMN) represents the SMO which consists of two actors (called lanes in BPMN). There is first of all a human on duty incident manager who performs monitoring tasks and acts as dispatcher for security incidents. The second actor is an enhanced GDSS that operates in a standby mode and that offers a library of process templates for security management processes.

Processes in BPMN are described mainly as flow (solid arrows with optional labels) of activities (labeled blue rounded boxes in Figure 2; a toothed wheel indicates that the completion is performed by some machinery) that can be controlled by so-called gateways (yellow diamonds). 
Gateways that split the flow into multiple outgoing flows with only one selectable option are called exclusive gate-ways (empty diamond), gateways that permit all possible combinations of outgoing flows are called inclusive gate-ways (indicated by “O”), gateways that force all outgoing flows to occur in parallel are called parallel gateways (in-dicated by "+").

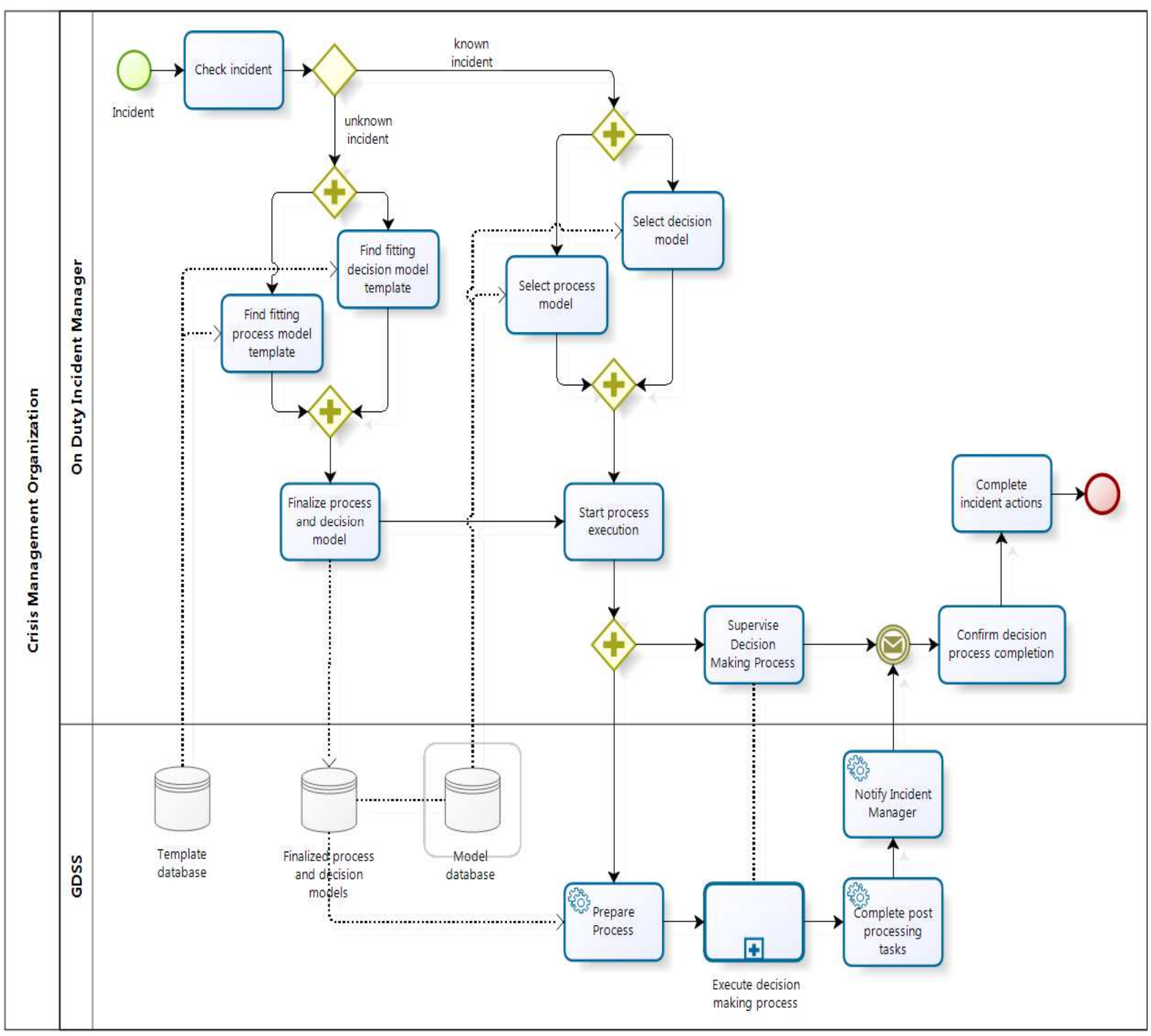

Figure 2. Security Incident Management Process.

Another major modeling primitive of BPMN are events (circles with optional labels). A start event (green circle) and an end event (red circle) are to be modeled for every process. BPMN also supports a set of other event types such as an intermediate message event (indicated by the envelope symbol).

In the sample process the initial activities target the goal to determine appropriate templates for the intended group decision making process and the needed underlying decision model. When the incident is not known the best fitting process template and best fitting group decision template are selected from a template database. This selection task can for example be supported by a proper decision tree. If necessary the selected templates within certain limitations can be adapted to the specific conditions of the given security management situation. Following that the incident manager starts a partially automated execution of the asynchronous group decision process in which the GDSS plays an active role. The activities performed by the GDSS are: prepare process, execute decision making process, complete post processing, and notify incident manager.

During the GDSS-based execution of the decision process the incident manager supervises the process and performs actions that are not automatically completed by the GDSS. The incident manager confirms the process completion when a corresponding completion message is received from the GDSS. In the last activity of the model the actions that are selected to handle the security situation are completed.

Note that there exists in the process model of Figure 2 an activity labeled execute decision making process that is 
marked with a little plus sign. This marking indicates that the details of this activity are modeled in an own process model in the form of a self-contained process. In general such refined model elements are referred to as sub-processes. The model of Figure 3 shows the process model of the sub-process Execute decision making process which cor-responds to an adaptive complete asynchronous group decision process. The pool consists of the three lanes referred to as Decision Moderator, GDSS, and Participants. Note that there is only a single lane does not mean that there only exists a single participant. The Decision Moderator needs not necessarily to be the same person as the incident manager. The two activities of the moderator are Make iteration decision and Revise process and/or decision model.

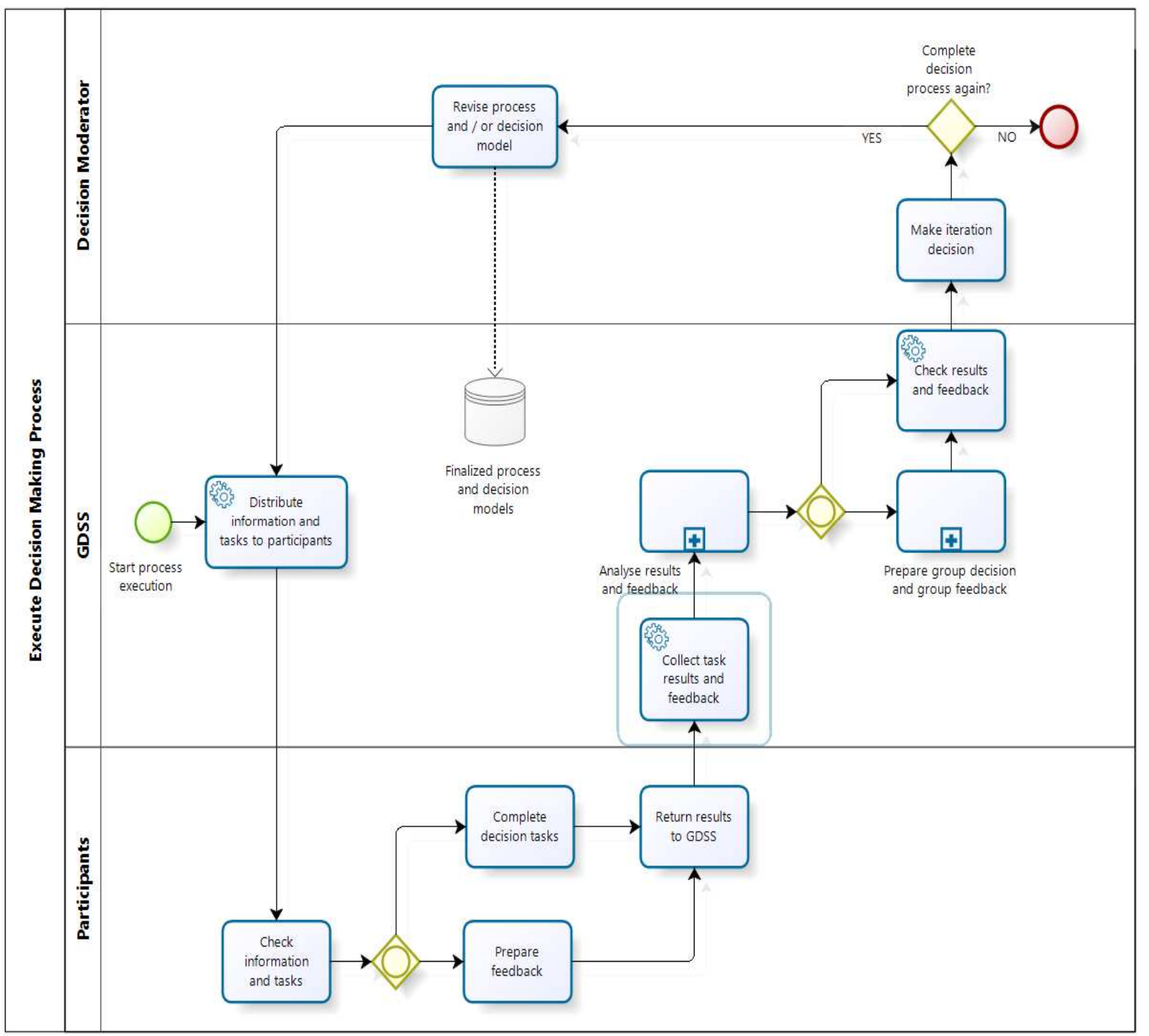

Figure 3. Adaptive Complete Asynchronous Group Decision Making Process.

The GDSS distributes the relevant information and deci-sion tasks to the participants. Furthermore, the GDSS collects the decision results and feedback, applies respective analysis such as consistency checks on the results and prepares the group result through an aggregation of the individual decision results. Note that the two respective sub-processes of the model of Figure 3 are not included in this article.

The participants check the decision tasks assigned to them by the GDSS and also the given decision relevant informa-tion. In turn they complete the decision tasks, prepare feedback that is useful for the securitysituation and the group decision, and return all results to the GDSS for further processing.

\section{Current Status and Conclusions}

The research focus has so far been on the conceptualiza-tion of the new adaptive group decision pattern and the development of a corresponding modeling approach using the BPMN modeling standard. Furthermore, also general functional enhancements for GDSS are developed and currently pioneered based on the GRUPO-MOD [17] re-search prototype. 
In the next steps the framework will be refined especially in two directions. A first refinement will address the issue if BPMN offers the expressive power that is needed to model all the relevant details and aspects that are important for group decision making processes. We will consider in this context the possibility to extend BPMN by new modeling concepts such as concepts for decision trees. Another direc-tion of future refinements will address technical integration issues and the required enhancements for GDSS. To auto-matically derive from a generic BPMN process model the respective runtime activities of a GDSS will be one of the major challenges. Furthermore, the required GDSS en-hancements to obtain and process feedback from the par-ticipants and to properly fuse and share the information present interesting challenges for future research. It is also planned to evaluate the results in terms of the bigger research framework that targets a combined use of process modeling techniques and GDSS for industrial security management. Therefore our future research agenda will also include corresponding laboratory and field studies.

\section{References}

[1] Bornstein, G., Kugler, T. andZiegelmeyer, A. (2004). Indi-vidual and group decisions in the centipede game: Are groups more "rational" players? Journal of Experimental Social Psychology, 40, 599-605.

[2] BSI-Standard 100-4e, Business Continuity Management, Version https://www.bsi.bund.de/SharedDocs/Downloads/EN/BSI/P ubli-

ca-tions/BSIStandards/standard_100-4_e pdf.pdf;jsessionid $=$ B516CCA64C402611062EA2AD7BFAA514.2_cid294? blob=publicationFile, accessed 11/23/2012.

[3] Comes, T., Hiete, M. and Schultmann, F. (2010). A decision support system for multi-criteria decision problems under severe uncertainty in longer-term emergency management, Proc. 25th Mini EURO Conference on Uncertainty and Ro-bustness in Planning and Decision Making, Coimbra.

[4] Esser, J. K. (1998). Alive and Well after 25 Years: A Review of Groupthink Research, Organizational Behavior and Human Decision Processes, Vol. 73, Nos. 2/3, pp. 116-141

[5] Fahland, D. and Woith, H. (2008). Towards Process Models for Disaster Response. Business Process Management Workshops, pp. 254-265.

[6] Gort, C. and Gerber, A. (2008). The Performance of Groups and Individuals in Financial Decision-Making, National Centre of Competence in Research Financial Valuation and Risk Management, Working Paper No. 460, February 2008, online

version:
http://www.ufsp.uzh.ch/finance/documents/WP460 A1.pdf, accessed 11/5/2012.

[7] Kapucu,N. and Garayev, V. (2011). Collaborative Deci-sion-Making in Emergency and Disaster Management, Taylor \& Francis Group, International Journal of Public Admin-istration, 34: 366-375.

[8] Levy, J.K., Hartmann, J., Li, K.W.,An,Y. and Asgary, A. (2007). Multi-criteria Decision Support Systems for Floor Hazard Mitigation and Emergency Response in Urban Wa-tersheds, Journal of the American Water Resources Associa-tion, April 2007, Vol. 43, No. 2.

[9] Mendonca, D., Rush, R., and Wallace, W.A. (2000). Timely knowledge elicitation from geographically separate mobile experts during emergency response, Elsevier Science, Safety Science 35, pp. 193-208.

[10] Object Management Group (2011): Business Process Model and Notation (BPMN) Version 2.0 (2011), OMG Document Number: formal/2011-01-03.

[11] Harand, A., Peinel, G. and Rose, T. (2011). Process Structures in Crisis Management, 6th Conference on Future Security, Berlin.

[12] Ramabrahmam, B. and Swaminathan, G. (2000). Disaster management plan for chemical process industries. Case study: investigation of release of chlorine to atmosphere, Journal of Loss Prevention in the Process Industries, 13, 1, 57-62.

[13] Saaty, T. L. (1980). The Analytic Hierarchy Process. McGraw Hill, New York.

[14] SANS Institute Glossary, http://www.sans.org/security-resources/glossary-of-terms/, accessed 11/21/2012.

[15] Song, Y. and Hu, Y. (2009). Group Decision-Making Method in the Field of Coal Mine Safety Management Based on AHP with Clustering, Proc. of 6th International ISCRAM Confe-rence, Gothenburg, Sweden.

[16] Stoner, J., (1961). A comparison of individuals and group decisions involving risks, Master Thesis, Massachusetts In-stitute of Technology, School of Industrial Management.

[17] Thimm, H. (2011). A System Concept to Support Asyn-chronous AHP-Based Group Decision Making, IADIS In-ternational Conference Collaborative Technologies, Rom, ISBN: 978-972-8939-40-3, pp. 29-38.

[18] Tseng, J., Liu, M., Chang, R., Su, J. and Shu, C. (2008). Emergency response plan of chlorine gas for process plants in Taiwan, Journal of Loss Prevention in the Process Industries, 21, 4, pp. 393-399.

[19] Turoff, M., Chumer, M., Van de Walle, B., Yao, X. (2004). The Design of a Dynamic Emergency Response Management Information System, Journal of Information Technology Theory and Application (JITTA), Vol. 5(4), pp. 1-36. 